\title{
Mujer indígena en la administración de recursos naturales en la comunidad de Francia Sirpi
}

\author{
Indigenous woman in the natural resource's administration in France Sirpi Community
}

\author{
Pilar Muller Oporta'
}

\section{Resumen}

El estudio ha tratado sobre la participación de las mujeres indígenas en la administración de los recursos naturales en la comunidad de Francia Sirpi. Describe los espacios y roles, identifica factores que limitan y posibilitan la participación de éstas dentro de los sistemas organizativos y de toma de decisiones, así como también valora su percepción sobre participación en la administración de los recursos naturales. El estudio fue cualitativo y cuantitativo. Describe situaciones, interacciones, actitudes y comportamientos observables; detalla datos estadísticos comprobables. Las técnicas aplicadas fueron entrevistas, observación participante, grupos focales, encuesta y revisión documental. Se encontró que las mujeres indígenas de la comunidad de Francia Sirpi, a pesar de estar involucradas en actividades dentro de la comunidad, tienen limitantes no sólo en la comprensión del término, sino en la interiorización de éste, para el ejercicio de sus derechos. Factores socioculturales y estilo de vida condicionan el acceso a modelos y sistemas organizativos para el ejercicio de derecho para la toma de decisión y administración de recursos naturales donde la participación de las mujeres indígenas se ha limitado sólo al uso y acceso a recursos naturales.

El estudio aporta a concientizar a estudiantes de sociología y carreras afines, a mujeres indígenas, a autoridades comunales, territoriales, municipales y regionales, sobre la importancia de la participación de la mujer en espacios para la toma de decisión y promover estrategias acordes con el contexto y con la realidad que se encuentran las mujeres indígenas de la región.

Palabras clave: recursos naturales; mujeres indígenas, administración.

\section{Abstract}

The study has dealt with the indigenous women participation in the administration of natural resources in France Sirpi community. It describes the spaces and roles, identifies factors that limit and enable their participation within the organizational and decision-making systems, as well as assessing their perception of participation in the natural resources administration.

The study was qualitative and quantitative. It describes situations, interactions, attitudes and observable behaviors; it details verifiable statistical data. The techniques applied were interviews, participant observation, focus groups, survey and documentary review.

It was found that the indigenous women from the France Sirpi community, despite being involved in activities within the community, have limitations not only in the understanding of the term, but in the internalization of it, for the exercise of their rights. Sociocultural factors and lifestyle influence the access to models and organizational systems for the exercise of law for decision making and the natural

\footnotetext{
1 Licenciada en Sociología con Mención en Autonomía por la Universidad de las Regiones Autónomas de la Costa Caribe Nicaragüese. Correo: pemotini7@ yahoo.com; ORCID: https://orcid.org/0000-0003-4146-4162
}

Recibido: 25/06/2018 - Aprobado: 24/01/2019 
resources administration where the participation of indigenous women has been limited only to the use and access to natural resources.

The study provides awareness to sociology students and related careers, indigenous women, communal, territorial, municipal and regional authorities, on the importance of women's participation in spaces for decision making and promote strategies according to the context and with the reality that the indigenous women of the region meet.

Keywords: natural resources; indigenous women, administration.

\section{Introducción}

Esta investigación tiene el propósito de conocer los mecanismos de participación de la mujer indígena en la administración de los recursos naturales. Se desarrolló de agosto a noviembre del 2010 en la comunidad de Francia Sirpi, territorio indígena Wangki Twi- Tasba Raya, comunidad del municipio de Waspam, Región Autónoma del Atlántico Norte, pueblo miskitu.

Se analiza la importancia que tiene la participación de las mujeres indígenas en la administración de los recursos naturales y el rol que desempeñan dentro de las estructuras organizativas comunales, formando parte de estas solo como líderes sectoriales (representantes de la organización de mujeres) y no en espacios de toma de decisiones. Se identifica una alta participación de mujeres indígenas en las actividades productivas y económicas, así mismo un bajo nivel en la toma de decisiones de las mujeres en cuanto a ocupar cargos de relevancia como el de "wihta" (juez) o síndico.

Se aportan resultados significativos a la hora de desarrollar en la comunidad nuevos proyectos, sobre equidad de género y manejo de recursos naturales, considerando la formación humana de las mujeres indígenas de mucha importancia para la administración de los recursos naturales.

\section{Revisión de literatura}

\section{Comunidad}

La ley $445^{2}$, arto, 3: define comunidad como el conjunto de familias de ascendencia amerindia establecidas en un espacio territorial, que comparten sentimientos de identificación, vinculados al pasado aborigen de su pueblo indígena y que mantienen una identidad y valores propios de una cultura tradicional, así como formas de tenencia y uso comunal de tierras y de una organización social propia.

\section{Participación de la mujer indígena}

La Convención para la eliminación de todas las formas de discriminación contra la mujer (CEDAW, 1979), reconocen los derechos fundamentales a favor de las mujeres; a la no discriminación, a la igualdad de derechos y oportunidad de participar y ocupar cargos en diferentes esferas sociales, a no ser sujetas de exclusión o restricción basados en el sexo.

La Constitución Política de Nicaragua a través dela Ley648, Ley de Igualdad de Derechosy Oportunidades (2008), establece en su artículo 8: "Los poderes del Estado, sus órganos de administración a nivel nacional, los Gobiernos de las Regiones Autónomas de la Costa Atlántica, las municipalidades y las instituciones de creación constitucional garantizaran la igualdad entre mujeres y hombres en el ejercicio de sus derechos políticos, incluidos entre otros, el derecho al voto, la elegibilidad, el acceso a las instancias, niveles de

\footnotetext{
2 Ley de Régimen de Propiedad Comunal de los Pueblos Indígenas y Comunidades Étnicas de las Regiones Autónomas de la Costa Atlántica de Nicaragua y de los ríos Bocay, Coco, Indio y Maíz.
} 
poder y toma de decisiones, así como la libertad para organizarse, de participar y demás garantías civiles y políticas".

La verdadera participación significa que las mujeres indígenas dentro de sus órganos de gobiernos comunales tengan espacios, la capacidad de expresar sus puntos de vista y tomar las decisiones concernientes a situaciones que afecten sus vidas.

\section{Mujer indígena y recursos naturales}

Es crucial la participación de las mujeres en los procesos de gestión de los recursos naturales, debido a que las mujeres se inclinan más que los hombres en la búsqueda de la integración de indicadores tangibles como la distribución económica, la transparencia y eficiencia en el manejo de fondos, así como indicadores intangibles relacionados a satisfacción de la colectividad y no solo de un grupo (Pandolfelli et al., 2007; Agrawal et al., 2006).

Generalmente las mujeres indígenas tienen acceso a determinados recursos como la pesca, el agua y los productos no maderables del bosque; pero solo el acceso no es suficiente, se requiere que ellas también participen en la toma de decisiones relacionadas a la distribución equitativa de los beneficios derivados del uso de estos (Pelusso y Ribot, 2003).

La participación de la mujer en la administración de recursos naturales en un tema relativamente nuevo en Nicaragua ya que estamos viviendo en una sociedad patriarcal en la que el enfoque de género está inclinado en el tema hacia los hombres en su mayoría.

Massolo (2006), Llanos y Sample (2008) expresan que el acceso de las indígenas al gobierno local comunitario les brinda legitimidad política, y con frecuencia mejora la gobernabilidad, dado que suelen empujar procesos de toma de decisiones más transparentes y participativos.

La FAO (2006) plantea que, dada su estrecha vinculación con los recursos naturales, las mujeres rurales son las más preocupadas por la conservación de los bosques, ya que de la naturaleza obtienen agua, medicina, arcilla, frutas y leña.

\section{Derechos de las mujeres indígenas a los recursos}

La Ley 648, Ley de Igualdad de derechos y oportunidades en su Arto 48, establece la igualdad incondicional de todos los nicaragüenses en el goce de sus derechos políticos; en el ejercicio de los mismos y en el cumplimiento de sus deberes y responsabilidades, aclarando categóricamente que existe igualdad absoluta entre el hombre y la mujer.

La Ley 28, Estatuto de Autonomía de las dos Regiones de la Costa Atlántica de Nicaragua (1987), en su Arto 11, inciso 1, Constitución Política de Nicaragua. Managua. Nicaragua, establece que los habitantes de las comunidades de la Costa Atlántica tienen derecho a la absoluta igualdad de derechos y deber, independientemente de su número poblacional y nivel de desarrollo; asimismo en el inciso 7 se les reconoce el derecho a elegir y ser elegidos, como autoridades propias de las Regiones Autónomas.

De igual manera el arto 23, inciso 14, atribuye al Consejo Regional promover la integración, desarrollo y participación de la mujer en todos los aspectos de la vida política, social, cultural y económica de la región. (La Gaceta, Diario Oficial No. 155 del 18 de agosto, 2016 


\section{Materiales y métodos}

La investigación es de carácter cualitativa y cuantitativa. Las técnicas definidas son las entrevistas abiertas, entrevistas a mujeres, grupos focales, y la observación participante. Los instrumentos fueron las guías de las técnicas señaladas, grabadoras, papelón y libreta de campo.

Para su desarrollo se tomaron en cuenta algunas variables: Espacios y Roles de Participación Comunitaria de las Mujeres Indígenas, Participación de la Mujer en el uso, acceso, producción agrícola y comercialización, Condicionantes que enfrenta la mujer Indígena para participar en los sistemas organizativos y de toma de decisión, Percepción de la mujer indígena sobre su participación. Para el desarrollo del trabajo se consideraron indicadores sociales, culturales y económicos.

El universo fue de mil, ciento treinta y ocho $(1,138)$ habitantes, de los cuales, cuatrocientos cincuenta y seis (456) son mujeres. La muestra se escogió por conveniencia: sesenta y ocho mujeres, de la comunidad de Francia Sirpi.

La información y datos obtenidos no serán utilizados para fines lucrativos, sino que tienen el propósito de que los resultados contribuyan para el desarrollo de mejores oportunidades y aporte para investigaciones futuras.

\section{Resultados y discusión}

El presente estudio sobre las mujeres indígenas en la administración de recursos naturales refleja los siguientes resultados:

\subsection{Participación de las mujeres indígenas de la comunidad de Francia Sirpi en la admi- nistración de los recursos naturales}

Los niveles para la administración y aprovechamiento de los recursos naturales identificados son: (i) La familia, la cual administra pequeñas parcelas o áreas asignadas por la autoridad comunal e internamente con sus miembros establece los mecanismos de usufructo de los recursos naturales en su posesión, (ii) La asamblea comunal, máxima expresión de toma de decisiones dentro de la comunidad, quien delega esta responsabilidad en el síndico y (iii) A nivel de los territorios recae en su asamblea quienes eligen al síndico territorial, como su representante en toda su área geográfica en posesión, este debe establecer mecanismos de coordinación con cada uno de los síndicos comunales que integran dicho territorio para implementar normas, acuerdos y responsabilidades de uso y distribución de los beneficios.

En la siguiente gráfica se aprecia los diferentes niveles de administración de recursos naturales y sus espacios de gobernanza comunitario y territorial.

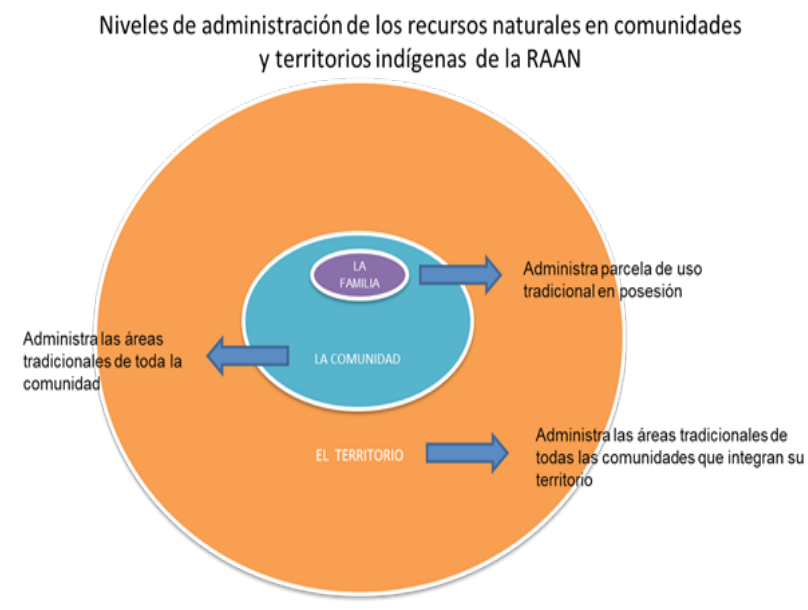

Figura 1. Niveles de administración de recursos naturales 
Las formas de administración de los recursos naturales a nivel de la comunidad presentan dos variaciones básicas; el (i) Usufructo con fines de autoconsumo, que realiza cada una de las familias en sus parcelas, las que fueron asignadas por la comunidad. Los productos generados dentro de estas áreas (granos básicos, tubérculos, raíces, musáceas, hortalizas, frutas), pueden ser comercializados, pero en cantidades relativamente bajas con los que se suplen algunas necesidades básicas dentro del hogar y estas son desarrolladas según el ciclo productivo al que corresponda. Dentro de esta categoría se incluyen los esfuerzos desarrollados para la caza, pesca, minería artesanal-familiar, aprovechamiento de madera (construcción de vivienda), (ii) El aprovechamiento con fines económicos se implementa cuando existe uso y aprovechamiento de recursos con la finalidad de generación de capital dentro de las áreas consideradas en posesión de la comunidad.

En ambos casos los síndicos comunales y síndicos territoriales deben de consultar a la asamblea comunal - territorial, regirse a las normas y acuerdos tradicionales establecidos para el usufructo de los recursos naturales y establecer los mecanismos de coordinación con los líderes sectoriales para facilitar la administración, monitoreo y seguimiento comunitario de los recursos naturales.

La siguiente gráfica representa la estructura para la administración de los recursos naturales en la comunidad de Francia Sirpi.

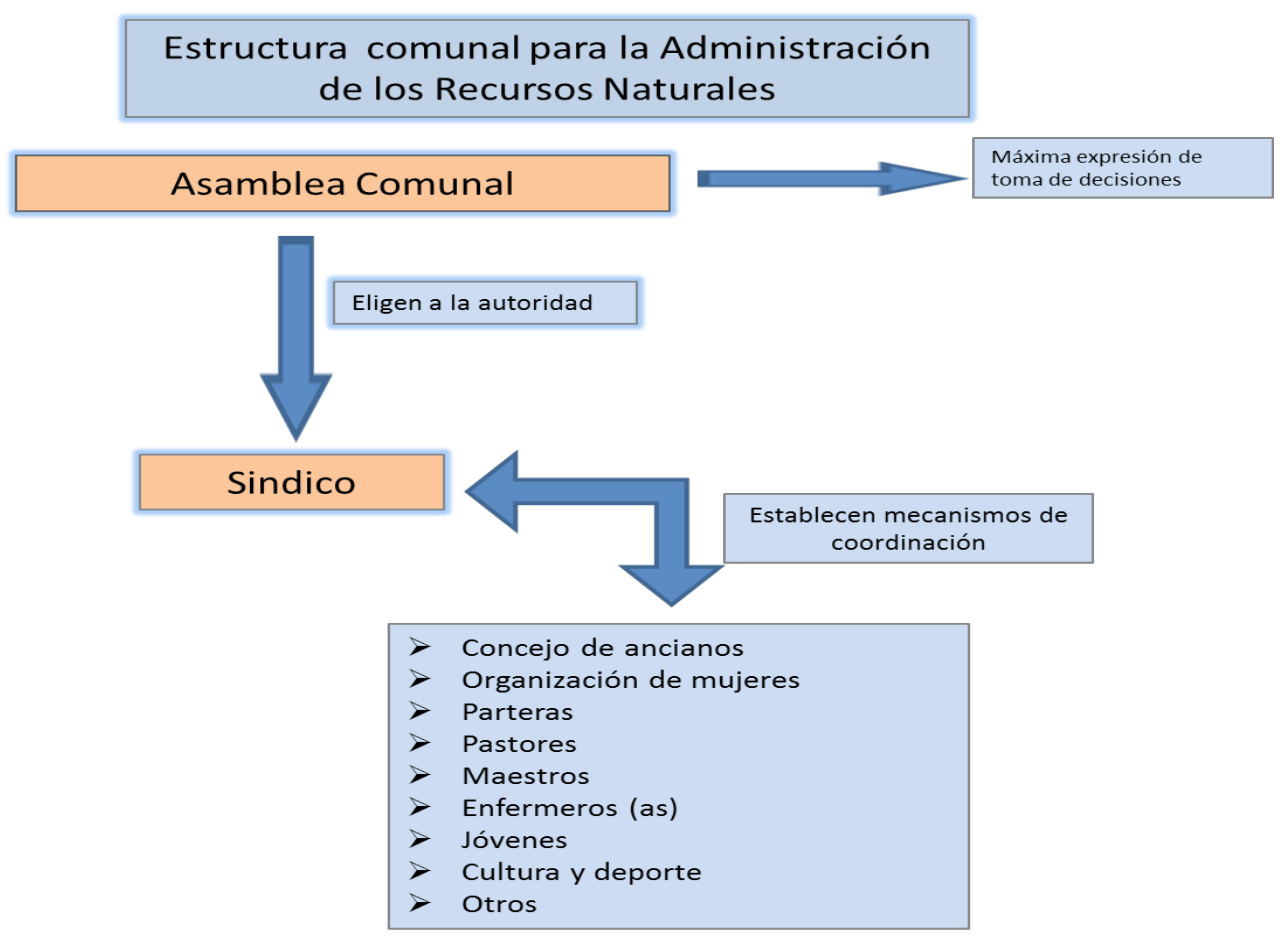

Figura 2: Estructura Comunal.

El análisis de las formas de organización comunitaria y territorial evidencia que dentro de estos órganos de administración y toma de decisiones son pocos los espacios de participación que tienen asignadas las mujeres. Para el primer caso (la comunidad) el sistema de selección y elección de sus autoridades en asamblea comunal, a través de una mayoría poblacional, aunada a factores socioculturales, intrínseco en sus formas y estilos de vida, conllevan a que las mujeres alcancen muy poco estos espacios de toma de decisiones (ser electas como autoridades: síndicos, wihta, coordinadora). 
Se reconoce que las mujeres se vinculan a muchas de las actividades económicas y productivas dentro de la comunidad entre las cuales se destacan el rol y acompañamiento que desarrollan junto con integrantes de cada uno de su familia en la realización de actividades vinculadas a prácticas agrícolas, forestales, recolección de frutos, elaboración y comercialización de carbón y leña, granos básicos, tubérculos entre otros, en las áreas asignadas a las familias dentro de la comunidad.

Los roles que han asumido las mujeres se han limitado a ser simples espectadoras de los procesos de administración de los recursos naturales donde en un periodo de 10 años, únicamente se logró identificar a dos mujeres que han ejercido algún tipo de cargo y desempeñado funciones dentro de la estructura organizativa de la comunidad, siendo esta la de coordinadora comunal (Entrevista lideresa, Francia Sirpi, 2010).

En este sentido se refleja un vacío de participación y acceso a la administración de los recursos naturales, a través de los mecanismos de organización comunitaria establecida. Aunque el cargo de coordinadora comunal forma parte de la junta directiva electa, en la práctica existe una relación de coordinación en la administración de los asuntos sociales y económicos internos de la comunidad y no necesariamente en la de los recursos naturales.

\subsection{Participación de la mujer en el uso, acceso, producción agrícola y comercialización de los recursos naturales}

Las mujeres indígenas que habitan en Francia Sirpi en su mayoría (90\% de las encuestadas) realizan y hacen uso de la diversidad de recursos naturales existentes dentro de su territorio con fines de autoconsumo (fundamentalmente; la agricultura, la pesca y recolección) y generación de ingresos económicos complementarios dentro de los hogares (comercialización de productos tales como; madera, artesanía y frutas).

La siguiente tabla detalla la participación de las mujeres indígenas de Francia Sirpi en el uso de los recursos naturales.

Tabla 1. Participación de las mujeres en el uso de los recursos naturales

\begin{tabular}{|l|l|l|}
\hline \multicolumn{1}{|c|}{ Uso } & \multicolumn{1}{c|}{ Actividad } & \multicolumn{1}{c|}{ Porcentaje } \\
\hline Recurso suelo (tierra) & Establecimiento de cultivos (frijoles, arroz, bastimentos) & $25 \%$ \\
\hline Recurso bosque & Elaboración de artesanía, recolección de leña y frutas & $25 \%$ \\
\hline Recurso Bosque (madera) & Construcción de viviendas y muebles rústicos para el hogar & $40 \%$ \\
\hline & Uso de agua y leña para el hogar & $10 \%$ \\
\hline
\end{tabular}

Fuente: Encuesta realizada a las mujeres indígenas, Francia Sirpi, sep. 2010

En la comunidad de Francia Sirpi como en todas las comunidades del territorio de Wangki Twi - Tasba Raya la principal fuente económica, es la agricultura. En ellas se cultiva frijoles, arroz, yuca, malanga, quequisque y musáceas, la cual es considerada de subsistencia debido a que la producción local es destinada para el autoconsumo (Grupo focal, Francia Sirpi, octubre 2010).

En la tabla que se muestra a continuación se detalla el involucramiento de la mujer en esta actividad.

Tabla 2. Participación de mujeres indígenas en Actividades Agrícolas

\begin{tabular}{|l|l|l|}
\hline \multicolumn{1}{|c|}{ Descripción } & \multicolumn{1}{c|}{ Si } & \multicolumn{1}{c|}{ No } \\
\hline Selección del cultivo & $75 \%$ & $25 \%$ \\
\hline Labores agrícolas & $100 \%$ & \\
\hline Preparación de los alimentos & $95 \%$ & $5 \%$ \\
\hline
\end{tabular}




\begin{tabular}{|l|l|l|}
\hline \multicolumn{1}{|c|}{ Descripción } & \multicolumn{1}{c|}{ Si } & \multicolumn{1}{c|}{ No } \\
\hline Preparación del suelo & $75 \%$ & $25 \%$ \\
\hline Siembras de cultivos & $100 \%$ & \\
\hline Selección de semilla & $80 \%$ & $20 \%$ \\
\hline Transporte de la cosecha & $95 \%$ & $5 \%$ \\
\hline Almacenamientos de la cosecha & $90 \%$ & $10 \%$ \\
\hline Comercialización & $75 \%$ & $25 \%$ \\
\hline
\end{tabular}

Fuente: Encuesta realizada a las mujeres indígenas, Francia Sirpi, sep. 2010

Con respecto a la participación de la mujer en las actividades agrícolas, las entrevistas opinan que las mujeres trabajan en todo el proceso de desarrollo agrícola, ellas son las que realizan: siembran, aplican fertilizantes, recolectan y trillan las cosechas. Ellas también trabajan en los cultivos secundarios, las legumbres y hortalizas, sin embargo, muy poco es reconocido el trabajo que realizan y por supuestos no es remunerado.

Con respecto a la actividad de pos cosecha (almacenamiento de la cosecha), la participación de la mujer representa el 90\%, porque en estas comunidades son las mujeres las generalmente recogen la cosecha, las mujeres aportan la mayor parte de la mano de obra necesaria para las actividades pos cosecha, responsabilizándose del almacenamiento, la manipulación, la constitución de reservas, la elaboración y la comercialización.

Las mujeres expresan que a pesar de participar en las actividades de producción trabajando a la par del hombre; es éste quien decide el rubro, el área de cultivo y la cantidad para consumo y comercialización de los productos. Sin embargo, destaca la participación de la mujer en la comercialización de bastimentos, frutas y en menor grado leña. El excedente de los productos obtenidos del bosque es comercializado en menor grado a nivel interno o en las cabeceras municipales de Waspam y Puerto Cabezas, en donde alcanzan mejores precios, pero condicionadas por dificultades de traslado (medios de transporte y época de lluvia) y regulaciones de precios que imponen los comerciantes de estos sectores.

El tema de comercialización de los derivados del bosque reviste particular importancia debido a que estos son realizados en su mayoría por hombres, quienes hacen mal uso de los ingresos que se generan (Resultado encuesta mujeres Francia Sirpi, 2010) por lo que los beneficios tangibles dentro de cada uno de los hogares son casi imperceptibles. En este sentido se reconoce que las mujeres son mejores administradoras de los ingresos económicos por lo que son ellas las que realizan las actividades de comercialización de los productos del bosque.

$\mathrm{Al}$ indagar por qué la mujer es la encargada de la comercialización, en términos generales, las mujeres argumentan que son mejores administradoras y que conocen las necesidades del hogar:

los hombres cuando van a Bilwi a vender invierten el dinero en cosas de menos importancia para el hogar y en algunos casos gastan en vicios y regresan a la comunidad sin ningún dinero y sin productos. (Grupo Focal, mujeres adultas, sept.2010)

\subsection{Factores que limitan a las mujeres indígenas en la organización comunitaria}

Existen factores que limitan la participación de las mujeres en el proceso de organización comunitaria, estos se vinculan en primera instancia con las prácticas culturales que son recreadas en los núcleos familiares en donde se considera que las mujeres tienen menos derechos que los hombres, por otro lado, los miembros varones de estas familias y compañeros de vida condicionan y limitan la presencia de sus conyugues e hijas en los espacios de selección y elección de autoridades comunitarias. 
Se aúna la poca confianza en sí mismas y nivel educativo que las mujeres tienen a lo interno de la comunidad para expresar y sostener sus opiniones y puntos de vista; en reuniones y asambleas comunales, así como el temor a no ser consideradas, tomadas en cuenta y reprimidas dentro de cada uno de sus hogares.

Otra limitante identificada es la marginación que cada una de las mujeres hace de ellas mismas, donde inicialmente se alega sobrecarga laboral dentro del hogar (cuido y atención de los hijos e hijas, actividades dentro del hogar: cocina, limpieza, lavado), especialmente cuando son madres solteras lo que dificulta que participe en los espacios de toma de decisiones dentro de la comunidad.

Asimismo, las mujeres consideran que asumir algunos cargos dentro de la junta directiva, las pone en condiciones de riesgos y optan por abstenerse. Otra de las consideraciones asociadas a lo anterior es que el ejercicio de algunos cargos dentro de las juntas directivas demanda tiempo, esfuerzo y dedicación dentro y fuera de la comunidad.

Como amas de casa no tenemos el tiempo, pues tenemos que atender la casa, al marido y los niños, eso de andar en el monte y resolver los conflictos es peligroso, no respetan a las mujeres (Grupo focal, mujeres adultas, Francia Sirpi, 2010).

Las últimas dos limitantes se fundamentan en una construcción social; que se ha venido recreando en la comunidad, en el que se considera a la mujer objetos y no sujetos de derecho con capacidades para asumir los diferentes roles y espacios dentro de la comunidad y en su junta directiva, así como la adopción y apropiación que las mismas mujeres han interiorizado de tener menor capacidad física e intelectual que el sexo masculino.

Sin embargo, a pesar de que en la actualidad existe en la comunidad mujeres organizadas, que trabajan, mujeres lideresas y líderes con la disposición de brindar espacios, en la práctica los datos de las encuestas arrojan datos de participación muy poco en temas relacionados a administración de recursos naturales.

Larson y Mendoza (2009) plantean que, en la Costa Caribe Nicaragüense, ha habido avances en la participación de las mujeres en los espacios de toma de decisiones en las estructuras de gobierno comunitario; pero a pesar de ello, en el territorio de Tasba Raya y en comunidades de Layasiksa, las mujeres indígenas continúan enfrentando limitaciones tanto en la participación en espacios de liderazgo a nivel comunitario, como en el acceso a los recursos forestales del territorio.

Esto se fundamenta con el estudio realizado por Smith (2010) quien asevera que la limitada participación de las mujeres mískitu del territorio de Tasba Raya en espacios de toma de decisiones y la falta de reconocimiento a sus capacidades de parte de los hombres, se debe a que el modelo de gobernanza indígena en el territorio de Tasba Raya, no ha logrado incorporar de manera particular las visiones y perspectivas de las mujeres.

Coincidiendo con los autores se demuestra que la principal causa de la poca participación es la falta de capacitación y el poco apoyo por parte de los líderes a nivel comunal y a nivel familiar por los compañeros de vida para incentivarlas a participar en estos espacios de toma de decisiones.

Las mujeres participamos muy poco en las actividades de la comunidad como reuniones y asambleas, así como también en espacios de toma de decisión porque somos amas de casa y vamos al monte a sembrar, tenemos que apoyar a nuestros maridos, también ellos (los hombres) necesitan más capacitación en el tema de equidad de género para abrir sus mentes y dar libertad a la mujer en sus casas para que asistan a las reuniones y para que participen en las reuniones y asambleas, así también los lideres tienen que invitar y motivar a las mujeres a participar (Comunitaria, Francia Sirpi, 2010). 


\title{
6.4 Factores que posibilitan la participación de las mujeres indígenas en el proceso de organización comunitaria
}

\author{
Posibilitan la participación en el proceso de organización comunitaria:
}

a). El interés común el cual se asocia con el establecimiento de pautas y criterios donde hay un beneficio individual y colectivo, donde se establezcan claramente los beneficios que se alcanzaran y a los cuales contribuirá esta alianza estratégica.

b). La formación, visibilización y reconocimiento de derechos a favor de mujeres y los procesos de acompañamiento desarrollados por diferentes organizaciones en la comunidad.

c). La distribución de roles y responsabilidades dentro del hogar y el apoyo de la familia permite que las mujeres dispongan del espacio y tiempo necesario para participar en reuniones, asambleas y espacios de concertación donde se seleccionan y nombran a los diferentes representantes u autoridades de la comunidad. La participación no se limita a un acto de presencia sino a uno en el que expongan las opiniones y puntos de vistas propios que en su conjunto hacen la fuerza (Grupo focal, Francia Sirpi, octubre 2010).

d). La necesidad de establecer sistemas de organización y representación propias de las mujeres en la comunidad lo que facilita y visibiliza sus demandas, necesidades y perspectivas para su desarrollo integral (cultural, social, económico, organizativo).

\subsection{Percepción de la mujer indígena sobre la toma de decisión para la administración de los recursos naturales}

La distribución de roles que se les ha impuesto a las mujeres indígenas implica en su forma de ver y sentir acerca de su participación en espacios de toma de decisión, pues desde niñas se les ha inculcado que deben realizar actividades específicas tales como las del hogar y los hombres las labores del campo, lo que les ha limitado la oportunidad de optar a cargos de relevancia dentro de las estructuras organizativas de la comunidad.

Massolo (2006), Llanos y Sample (2008) expresan que el acceso de las mujeres indígenas al gobierno local- comunitario les brinda legitimidad política y con frecuencia mejora la gobernabilidad, dado que suelen empujar procesos de toma de decisiones más transparentes y participativas.

Lo expresado por los autores difiere de la realidad de la comunidad de Francia Sirpi, ya que a pesar de que el espacio "existe", las mujeres no se postulan a ocupar los mismos. En este sentido, una preocupación particular de las mujeres es el acceso al conocimiento para fortalecer sus capacidades. La participación efectiva de las mujeres se dará si ellas están preparadas y con el acceso a la información y toma de decisiones, para ellas es fundamental la capacitación permanente considerando todos aquellos aspectos que le obstaculizan dicha participación en estos tipos de actividades.

Como condiciones necesarias para una mayor participación de las mujeres indígenas en fortalecer sus conocimientos e incidir en la toma de decisiones, dentro y fuera, de sus comunidades, las mujeres plantearon la ayuda del esposo en el cuidado de los hijos, para poder participar mejor, mayor atención y respeto de parte de las autoridades en las asambleas comunales, el reconocimiento de parte de los hombres de su capacidad de pensar y emitir opiniones.

A pesar de que el rol de las mujeres indígenas en la vida comunitaria está relacionado a la transmisión de conocimientos cosmogónicos y culturales, las mujeres que viven en esta comunidad han desempeñado tradicionalmente un papel muy limitado en el proceso de toma de decisiones. Las concepciones tradicio- 
nales sobre el lugar de la mujer en la sociedad y la falta de igualdad de oportunidades en el acceso a la participación son dos de los numerosos obstáculos a su participación.

Una característica del grupo miskitu es el machismo, que se refleja por medio de la discriminación hacia la mujer a quien se le considera como una obligación el trabajo doméstico y a su vez la falta de opción a cargos de líderes tradicionales.

"Tenemos que seguir luchando contra la discriminación, que los hombres sepan que nosotras tenemos capacidad también ese es nuestro reto". (Grupo Focal, oct. 2010)

En este sentido desde la percepción de las mujeres indígenas se vislumbra el deseo de abrir brechas e ir cambiando la mentalidad de los hombres y de ellas mismas en la comunidad y la necesidad de unirse para lograr los objetivos ni apoyo entre las mismas mujeres quienes son las que deben proponer a uno de sus miembros, pero al no sentir respaldo no optan a cargos. "Nosotras las mujeres estamos buscando los espacios entre los hombres, pero se ve que no hay unidad entre las mujeres." (Grupo focal, mujeres jóvenes, Francia Sirpi, 2010). Una de las opciones que las mujeres sienten fortalecería tanto a la organización de mujeres como a las comunitarias, es el de contar con un local, un espacio de concertación dentro de la comunidad, mismo donde se brinde asesoría en temas de liderazgo y enfoque de género. "Debemos de buscar espacios para hablar de nuestras cosas, fortalecernos y ser más fuertes no para luchar contra los hombres sino demostrar cómo somos capaces, recordemos que la mujer busca para sus hijos a como sea" (Grupo focal, mujeres adultas, Francia Sirpi, 2010).

La percepción de las mujeres indígenas de la comunidad es que necesitan demostrar que ellas también pueden ocupar cargos de relevancia y de toma de decisiones sobre los recursos naturales, así como Wihtas o síndicos.

\section{Conclusiones y recomendaciones}

La participación comunitaria desde la óptica de las mujeres indígenas de Francia Sirpi, evidencia que existe un vacío, no sólo en la comprensión del término, sino en la interiorización que tienen las mujeres para el ejercicio de sus derechos fundamentales dentro de la comunidad que deben de trascender de hacer acto de presencia hacia otros en donde se construyan propuestas, mecanismos de planificación, desarrollen estrategias de concertación y negociación para el acceso no solo al uso de los recursos naturales, sino a la administración de ellos.

La exclusión y autoexclusión por diferentes factores socioculturales y estilos de vida condicionan el acceso a los modelos y sistemas organizativos internos para el ejercicio de administración de los recursos naturales a nivel de la comunidad.

Una de las principales limitantes es que la administración de los recursos naturales es concebida por las mujeres de la comunidad (grupo focal, mujeres adultas, noviembre 2010), como el uso y aprovechamientos que realizan de los bienes y servicios que pueda brindar la naturaleza (aprovechamiento forestal, medicina tradicional, recolección de frutos, prácticas agrícolas, caza, pesca) y no como el ejercicio de regulación y normación del usufructo a nivel comunitario.

Por otro lado, la poca articulación interna entre todas las mujeres que habitan en la comunidad condiciona que estas puedan hacer propuestas, generar consenso y nombrar a uno de sus miembros para que ocupen algunos de los cargos dentro del sistema organizativo de la comunidad y especialmente para la administración de los recursos naturales.

La participación de la mujer en el uso de los recursos naturales ha sido de relevancia pues esta asume roles que sustentan el desarrollo económico y social de la comunidad. Es así como apoyan tanto en las 
labores agrícolas, cultivo de siembras, actividades post cosecha y comercialización de los productos, así como también de las labores del hogar, haciendo el papel de madres, esposas y agricultoras.

Las mujeres indígenas de Francia Sirpi, no están empoderadas, no conocen sus derechos humanos como mujeres, no están relacionadas con sus cuotas de espacio y poderes en cuanto a sus liderazgos, no tienen apoyo de las instituciones ni espacio en la participación de toma de decisiones; sin embargo estas han empezado a conocer acerca de sus derechos y actualmente están en un proceso de asimilación de ellas, pues aún se sienten inseguras y con temor para hacerlos valer, esto les garantizaría su participación en la toma de decisiones en la administración de los recursos naturales.

\section{A manera de recomendaciones:}

a. En la organización comunitaria. Establecer un sistema de planificación entre los líderes territoriales y coordinadores de programas y proyectos para que establezcan una distribución de actividades en donde se incluya la participación activa y efectiva de la mujer en espacios y en los diferentes procesos de organización comunitaria que faciliten su inserción en la toma de decisiones para la administración de los recursos naturales e incorporación del enfoque de género y distribución equitativa para el desarrollo de la comunidad. Como un mecanismo de fomento y consolidación de participación de las mujeres dentro de la comunidad se hace necesario que dentro de las asambleas comunales se facilite un espacio para que estas expongan sus diferentes problemáticas y contribuyan de forma colectiva a la solución de las mismas y se establezcan mecanismos de seguimiento y monitoreo para verificar su cumplimiento.

\section{b. En la organización de mujeres:}

1. Se debe fortalecer las estrategias y bases de la organización a través de sistemas de planificación de trabajo que integre visiones y percepciones en común, incidencia, integrar a más socias, promover la participación.

2. Generar capacidad de hacer incidencia hacia las autoridades mismas, reconociendo sus derechos y partiendo de sus propias necesidades más sentidas.

3. Que las mujeres puedan contar con un local dentro de la comunidad que sirva de espacio de concertación y articulación de propuestas entre mujeres con la comunidad y actores externos.

\section{c. A los Organismos e Instituciones:}

Fortalecer la formación y capacitación en:

1. Sistemas organizativos

2. Incidencia y concertación para el desarrollo

3. Mecanismos de participación

4. Administración: organización comunitaria con enfoque de genero

5. Género y enfoque

6. Manejo forestal

7. Promover actividades de fortalecimiento orientadas a las mujeres para incrementar la participación de la producción agrícola y reforestación. 
8. Impulsar mecanismos alternos que contribuyan a que la mujer participe activamente en el comercio de subproductos que se obtienen del bosque, para mejorar los ingresos económicos de las familias.

9. Priorizar como áreas estratégicas de cambios la de educación y capacitación, incluyendo la formación y concientización en derechos de las mujeres.

10. Hacer esfuerzos enfocados a identificar nuevas oportunidades productivas y de mercado, y a apoyar la comercialización.

11. Que el gobierno municipal asegure la participación de las mujeres en las estructuras de la organización comunitaria para incidir en los espacios para la toma de decisión.

12. Promover las inversiones para el establecimiento de actividades económicas para las mujeres basadas en el uso de los recursos naturales, asegurando su sostenibilidad ambiental y el retorno económico de las mismas.

13. Diseñar políticas financieras en las instancias autonómicas que permitan el acceso de mujeres a un financiamiento digno.

\section{Lista de referencias}

La Gaceta (13 de diciembre 2002). Ley 445 Ley de régimen de propiedad Comunal de los pueblos indígenas y comunidades étnicas de las Regiones Autónomas de la costa atlántica de Nicaragua y de los ríos Bocay, Coco, indio y Maíz.

La Gaceta $\mathrm{N}^{\circ} 51$ (12 de marzo, 2008). Ley de igualdad de derechos y oportunidades.

La Gaceta, Diario Oficial (No. 155 del 18 de agosto, 2016). Ley no. 28, "Estatuto de Autonomía de las Regiones de la Costa Caribe de Nicaragua" con sus reformas incorporadas.

Larson, A. \& Mendoza, J. (2009.) Desafíos en la tenencia comunitaria de bosques en la RAAN de Nicaragua. Managua. URACCAN.

Llanos, B \& Sample, K (2008). Participación Política en América Latina. IDEA- Internacional. Lima, Perú

Massolo, A. (2006). Participación política de las mujeres en el ámbito local en América Latina, UNINSTRAW/ AECID. Santo Domingo, República Dominicana.

Pandolfelli, Lauren, Stephen Dorhn \& Ruth Maizen (2007). Gender and collective action. Policy implications from recent research en. CAPRI policy brief number 5

Smith, J. (2010). Mujer indígena y territorialidad: desafíos de equidad en la gobernabilidad de las Comunidades Indígenas de Tasba Raya. 